\title{
Predicting Postnatal Psychological Distress in a Zambian Cohort: Relationship between the EPDS and the GHQ-12
}

\author{
Lonia Mwape*, Kestone Lyambai, Kabwe Chitundu \\ University of Zambia, School of Nursing Sciences, Lusaka, Zambia \\ Email: *loniamagolo@gmail.com
}

How to cite this paper: Mwape, L., Lyambai, K. and Chitundu, K. (2019) Predicting Postnatal Psychological Distress in a Zambian Cohort: Relationship between the EPDS and the GHQ-12. Open Journal of Psychiatry, 9, 39-52.

https://doi.org/10.4236/ojpsych.2019.91004

Received: September 18, 2018

Accepted: January 26, 2019

Published: January 29, 2019

Copyright (c) 2019 by author(s) and Scientific Research Publishing Inc. This work is licensed under the Creative Commons Attribution International License (CC BY 4.0).

http://creativecommons.org/licenses/by/4.0/

\begin{abstract}
Postnatal mental distress is a public health problem and women screening positive of psychological distress who do not meet the diagnostic criteria for psychiatric disorder may be experiencing significant distress for which they might require assistance from mental health personnel. The risk of a woman experiencing psychological distress during the postnatal period can be predicted before it occurs. The study included 300 pregnant women who were selected systematically and on whom two assessments were conducted at two different points. The first assessment was conducted at 32-week gestation while the second one at six weeks following childbirth. The participants were assessed using the Edinburgh Postnatal Depression Scale (EPDS) and the General Health Questionnaire (GHQ-12). Analysis of Variance (ANOVA) was used to test the association between the EPDS and the GHQ-12 while logistic regression analysis was carried out in order to predict postnatal psychological distress. The results showed no significant difference between the socio-demographic characteristics and the EPDS score. A significant association was found between the GHQ-12 and EPDS scores ( $r=0.545 ; \mathrm{p}=0.001)$. Logistic regression revealed that a single predictor, antenatal EPDS individual score, alone provided a highly adequate model and was statistically reliable in distinguishing between presence and absence of psychological distress in the postnatal period $(-2 \log$ likelihood $=225.634$; CI (1.035 - 1.173); X2 (1) 9.633, $\mathrm{p}=0.002)$. The study revealed a correlation between EPDS score and GHQ-12 and that the EPDS can predict postnatal psychological distress using the data from the antenatal period. Therefore, patients who are found with antenatal psychological distress should be consistently evaluated throughout pregnancy and during the postnatal period to allow for early detection and intervention of postnatal psychological distress.
\end{abstract}




\section{Keywords}

Psychological Distress, Antenatal, Postnatal, EPDS, GHQ-12

\section{Background Information}

Psychological distress is a leading cause of disease-related illness among women in general and postnatal women in particular, and its prevalence has been estimated to be higher compared to men [1]. Most of it is under reported in many resource-constrained societies such as sub-Saharan Africa [2]. Postnatal mental distress is a public health problem and women screening positive of psychological distress who do not meet the diagnostic criteria for psychiatric disorder may be experiencing significant distress for which they might require assistance from mental health personnel [3] [4].

The risk of a woman experiencing psychological distress by postnatal women can be predicted before it occurs [2] [5]. Although there is no universally accepted method for identifying women who may be experiencing a mental health problem, it is recommend that pregnant women are screened and assessed for psychological distress in the perinatal period to facilitate early problem identification and intervention [2] [6] [7]. The Edinburgh Postnatal Depression Scale (EPDS) is a simple, safe and important screening and diagnostic tool for perinatal psychological distress [8]. The GHQ-12 is another screening tool which has also been extensively used as a short screening instrument for psychological distress in a variety of clinical settings [9].

The psychological distress related to motherhood can be experienced any time during either the antenatal or postnatal period or both. The prevalence rates for the mentioned periods may be similar to those in the general population and range between 12 and 20 percent in high-income countries, and slightly higher in low and middle-income settings [6] [10] [11]. Women in the perinatal period may experience an array of emotions including joy and occasional despondency. Some mothers may experience episodes of "the baby blues" as hormonal changes stabilize. However, the mood swings associated with baby blues are not a psychiatric disorder, and mostly resolve within a couple of weeks [12].

Unidentified or untreated psychological distress may culminate in Postnatal Depression (PND), an incapacitating condition where the patient presents with persistent low mood. Postnatal Depression affects between 5 - 15 percent of mothers in high-income settings and a higher percentage in low and middle-income settings [2] [5]. The high prevalence rate in low and middle-income countries is attributed to low health seeking behaviours by women [13]. The consequences of perinatal psychological distress are extensive and may affect the mother, her infant, their relationships, and the community as a whole.

In pregnancy, psychological distress may reduce the mother's ability to care for herself in terms of maintaining nutrition, and antenatal clinic attendance, 
which may jeopardize a woman's physical mental health and diminish ideal foetal monitoring, culminating in compromised growth and development of the baby [14] [15].

It is well documented that children born to psychologically distressed mothers often tend to be underweight, stunted and have impaired social and cognitive development [16] [17]. Further, the interactional relationship between mother and baby may be compromised in the presence of postnatal depression, the effects of which may be of greater detriment to the child, although the impact on child development is quite uncertain especially in high socioeconomic populations where the impact is said to be greater when postnatal depression is chronic and severe [18].

Räisänen et al. [19] reveals that mothers with a history of previous psychological distress are at high risk of developing psychological distress in the postnatal period, although in some cases, it has been a challenge to predict psychological distress risk in women without a previous history. Therefore, history of psychological distress is usually the most certain risk factor predisposing a mother to developing PND. This notwithstanding, mothers without previous episodes of psychological distress are at increased risk of psychological distress if they experience stressful events in the antenatal period or during delivery. Such events may include but not limited to caesarian section, premature birth or congenital malformation of the baby [19]. Interestingly, uncertainty about the upcoming childbirth has also been found to be a strong predictor of psychological distress [19].

Considering the high prevalence and serious consequences of antenatal and postnatal psychological distress and depression, deliberate efforts should be made to identify risk factors that may guide the plans for interventions. Subsequently, identifying women who are at higher risk of psychological distress in the antenatal period may facilitate prediction of psychological distress in the postnatal period [14].

However, little work has been carried out on predicting postnatal psychological distress and previous work have not comprehensively considered addressing the Predicting Postnatal Psychological Distress in a Zambian Cohort and looked at the relationship between the EPDS and the GHQ-12.

Therefore, this paper seeks to predict postnatal psychological distress using data obtained during the antenatal period and show the ability of the EPDS to yield similar results with the GHQ-12 in detecting postnatal psychological distress on one hand, and its ability to predict postnatal psychological distress using data from the antenatal period, on the other hand.

\section{Methods}

The paper is based on a study that was conducted on a Zambian cohort of women during the antenatal and postnatal periods. The data was collected over a period from May to August 2017 using the Edinburgh Postnatal Depression 
Scale (EPDS) and the General Health Questionnaire GHQ12. The EPDS [20] is a ten item self-report screening tool for depression that has been used widely postnatally and has also been validated for use antenatally (Cronbach's alpha 0.77). The instrument has been suggested to be measuring both anxiety and depression in the last trimester of pregnancy and also in postnatal stages [21]. Each item on the EPDS is scored on a four-point scale ranging from 0 to 3, starting with either the highest score (3) or the lowest score (0), and giving a total score of 30 [20]. According to Eberhard et al. [22], the EPDS has been validated in a number of situations as a screening instrument for postnatal depression. Although the EPDS in itself cannot confirm the diagnosis of postnatal depression, a score of over 12 is considered to indicate the possible presence of postnatal depression. Adjustment of the EPDS cut off point also allows for identification of psychological distress. Hence, Brouwers et al., [23] and Ross et al., [24] recommend the tool as psychometrically reliable for use throughout the perinatal period.

On the other hand, the General Health Questionnaire (GHQ-12) [25] is a twelve-item instrument designed to screen for psychological disturbance in a wide variety of clinical groups. It is the shortest among the five versions (GHQ-12, GHQ-28, GHQ-30, GHQ-36, and GHQ-60). The GHQ-12 has been recommended as an accurate measure of psychological distress in all clinical populations because of its design as a self-report questionnaire that is easy to administer and score. In recent years the GHQ-12 has been extensively used as a short screening instrument [9]. Goldberg et al. [26] reveals that the GHQ-12 works as effectively in low-income countries as it does in elsewhere.

The EPDS and the GHQ-12 were used on a sample of 300 women in the third trimester of pregnancy ( 32 weeks) while 198 of the same women were interviewed during the first six weeks following childbirth. The sample size was determined using the Dobson formula assuming a prevalence of postnatal mental distress among women to be 25 percent. The calculated sample size obtained was adjusted to 300 assuming a 10 percent non-response.

Ethical approval was received from Leeds Metropolitan University and from the University of Zambia Biomedical Research Ethics Committee. The Lusaka District Health Management gave clearance to the research team to access to health facilities that were included in the study. Respective facility managers gave permission to access participants. The purpose of the study was explained to the participants and information concerning participation and consequences of the study was availed to all them. Anonymous and confidentiality were upheld, but it was made clear to the participants that the information they volunteered would be shared with medical personnel should it be established that they were at risk. Participants were informed of their right to withdraw from participation any time without prejudice. Those who were willing to participate then provided consent prior to the interview.

The data were analysed using the Statistical Package for Social Sciences (SPSS) 
version 21.0. The sequence of the analysis for both the antenatal and postnatal phases of the study were the same except for measures of central tendency on demographic characteristics of participants which were carried out only for the antenatal phase and could not be repeated in postnatal phase considering that participants were the same for both phases.

Association between age, parity, marital status, educational level, employment status, and residential area was explored using the Analysis of Variance (ANOVA), except employment status on which the t-test was used. Post Hoc Tests were then conducted in order to make multiple comparisons. Finally, logistic regression analysis was carried out in order to predict postnatal psychological distress using antenatal data. This involved recoding the dependent variable (postnatal EPDS score) to make it a dichotomous (present of psychological distress, and absence of psychological distress) before running the analysis. Three variables (Antenatal EPDS, GHQ-12 score) were entered as independent variables used to predict the dependent variable.

\section{Results}

\subsection{Demographic Characteristics}

A total of 300 women in their third trimester of gestation (32 weeks gestation) were enrolled to participate in the study. The age ranged between 18 and 45 years, with the mean age of 27 years and Standard Deviation of 5.9. The median age was 27 where according to Mofrad et al. [27], expectant mothers have greater risk of psychological distress and other perinatal problems. The largest number of participants concentrated within the age of 20 - $34(\mathrm{n}=206)(70.1 \%)$ were not employed compared to $\mathrm{n}=49(16.7 \%)$ who were in employment. The Central Statistical Office (CSO) [28] estimates employment rates as 50 percent for females and 79 percent for male, showing few women in employment compared men.

Most of the study participants $\mathrm{n}=250(83.3 \%)$ were married. According to CSO [28] figures, 60.2 percent of women in Zambia were married. However, this number does not include those who are cohabiting with or have separated from their spouses. The majority of the participants $n=254(83.3 \%)$ had either basic education (secondary and primary) or no education at all $n=17$ (5.7\%). CSO [28] estimates that only 21 percent of women between ages $20-29$ had completed secondary education compared to 45 percent of their male counterparts.

The study revealed that 146 of 300 women (48\%) had depressive symptoms. Out of this number, sixty women (20\%) had severe postnatal mental distress. Exploration of the association between the EPDS score and the social demographic characteristics of participants (age, parity, marital status, educational level, employment status, residential area) was explored using the Analysis of Variance (ANOVA), except employment status on which the t-test was used. Results suggest no relationship between any of the variables and the EPDS score. Post Hoc Tests were then conducted in order to make multiple comparisons. 
Finally, logistic regression analysis was carried out in order to predict postnatal psychological distress using the data collected in the antenatal period.

\subsection{Concurrent Validity-Antenatal EPDS, GHQ-12}

The EPDS has been validated in identifying antenatal and postnatal psychological distress and depression. Concurrent validity which refers to correlation between instruments was conducted between the EPDS, and the GHQ-12, and was measured using a correlation matrix to show the relationship between the two measures. Concurrent validity is often quantified by the correlation coefficient between two sets of measurements obtained for the same target population [29]. This involves the measurement performed by the evaluating instrument and by the standard instrument. In this case the evaluating instrument was the GHQ-12 while the standard instrument was the EPDS. This analysis was carried out in order to explore the possibility that the EPDS will yield similar results to those of the GHQ-12. Therefore, this suggests that the two different measurement tools used for the same population yield valid results.

Table 1 shows correlations between the two measures of psychological distress used in the present study (EPDS and GHQ-12). A significant association is evident between the EPDS and the GHQ-12 $(r=0.545 ; \mathrm{p}=0.001)$.

\subsection{Concurrent Validity-Postnatal EPDS and GHQ-12}

As earlier stated, concurrent validity between the EPDS, GHQ-12, was measured using a Pearson Correlation to show the relationship between the two measures shown above. This was in order to validate the EPDS for use in the population under study.

The correlation matrix (Table 2) shows correlation between the two measures of psychological distress used in the present study for the postnatal period (EPDS and GHQ-12). A relationship between the EPDS the GHQ is not significant.

Table 1. Antenatal EPDS, GHQ-12, SDIS correlation matrix.

\begin{tabular}{|c|c|c|c|c|}
\hline & \multicolumn{2}{|c|}{ EPDS individual score (antenatal) } & \multicolumn{2}{|c|}{ GHQ-12 individual score (antenatal) } \\
\hline & $(\mathrm{r})$ & $\mathrm{P}$ & $(\mathrm{r})$ & $\mathrm{P}$ \\
\hline EPDS individual score & - & - & 0.545 & 0.000 \\
\hline GHQ-12 individual score & 0.545 & 0.000 & - & - \\
\hline
\end{tabular}

Table 2. Postnatal EPDS and GHQ-12 correlation matrix.

\begin{tabular}{|c|c|c|c|c|}
\hline & \multicolumn{2}{|c|}{ EPDS individual score (antenatal) } & \multicolumn{2}{|c|}{ GHQ12 individual score (antenatal) } \\
\hline & $(\mathrm{r})$ & $\mathrm{P}$ & $(\mathrm{r})$ & $\mathrm{P}$ \\
\hline EPDS individual score & - & - & -0.100 & 0.159 \\
\hline GHQ-12 individual score & 0.100 & 0.159 & - & - \\
\hline
\end{tabular}




\subsection{Logistic Regression Analysis}

Forward logistic regression analysis was conducted to determine which variables among the two that were entered into the model (Antenatal EPDS and GHQ-12 scores) were predictors of postnatal psychological distress (presence, and absence of psychological distress). Regression results showed that a single predictor, antenatal EPDS individual score, alone provided a highly adequate model. It was statistically reliable in distinguishing between presence and absence of psychological distress in the postnatal period $(-2 \log$ likelihood $=225.634$; CI $(1.035$ - 1.173); X2 (1) 9.633, p = 0.002). The model correctly classified 97.9 percent of the cases. Regression coefficients are presented in Table 3.

Wald statistics indicated that the EPDS significantly predict psychological distress. However, Odds ratio for this variable indicated little change in the likelihood of developing psychological distress. The other variable (GHQ-12) could not predict postnatal psychological distress as illustrated in Table 3.

The discrepancy in prevalence and types of mental health problems may be attributed to methodological limitations, including small sample sizes, differences in tools used and heterogeneous definitions. Further to this, many of the studies of mental health in HIV positive adolescents conducted to date lack comparison groups and are mainly cross sectional, which makes it difficult to compare the prevalence of mental health challenges in this population to the general population. There is therefore urgent need to conduct longitudinal comparison studies to help draw conclusions about the prevalence of mental health problems and the potential contribution of HIV on the observed rates of mental health problems.

\section{Discussion}

The Edinburgh Postnatal Depression Scale (EPDS) is one of the commonly used screening tools for perinatal depression. It was initially designed to assess for postnatal depression but is currently recommended for use in the antenatal period as well [21]. The present study examined the ability by the EPDS to yield similar results with the GHQ-12 in detecting postnatal psychological distress as well as its ability to predict postnatal psychological distress using data from the antenatal period.

Table 3. Regression coefficients.

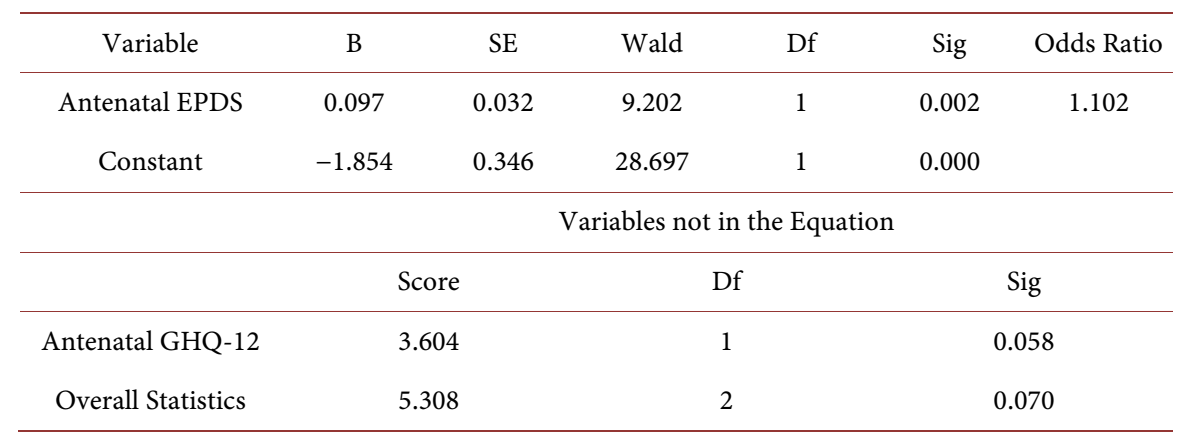


The EPDS was found to yield similar results with the GHQ-12 in the antenatal period. The mean scores of 9.2 and 10.4, for the EPDS, GHQ-12, respectively were found. Results from the postnatal period showed a wide difference between the mean scores for EPDS and the GHQ-12 (4.8 and 11.6 for the EPDS and the GHQ-12 respectively). Further, exploration of the association between the EPDS, GHQ-12, using Pearson correlation ( $r$ ) for both antenatal and postnatal data revealed an association between the EPDS and the GHQ-12 results ( $\mathrm{p}=$ 0.001 ) in the antenatal period.

Using instruments that possess the ability to measure what they are intended to measure is valuable to the health of all concerned. However, during the perinatal period, commonly used measures of psychological distress that have been validated for use in various clinical groupings may be psychometrically compromised despite previous findings [9].

The EPDS is a rough and ready means for identifying women in the postnatal period, who may require interventions for psychological distress [30]. The validity of the EPDS in identifying psychological distress during the perinatal period has been reported worldwide. The scale has been found to be suitable for use in non-postnatal women [31]. It is further asserted that chances that a woman scoring below the cut-off on the EPDS would be categorised as depressed are minimal because levels of depressive symptoms in a given person should be taken as just one of the factors that should be examined to assist in arriving at a decision on whether or not an individual requires referral to other professionals for therapeutic intervention [30]. If on the other hand, a higher cut-off is chosen, some women who might otherwise be clinically depressed or progressing to levels of disorder might be missed [30]. This explanation influenced the choice of a lower EPDS cut-off for the present study so that the chances of missing those women who may be experiencing psychological distress are be minimised.

On the other hand, the GHQ-12 has been recommended as a valid and reliable measure of psychological distress in all clinical groups. It possesses the ability to identify those experiencing probable non-psychotic psychiatric disturbance. The GHQ-12 has the precision to separate "cases" from "non cases" [26] and has been endorsed for comprehensive utility [32]. Lee et al. [33] writing from the United Kingdom recommends the GHQ-12 as accurate for screening for psychological distress in the perinatal period.

In contrast with Lee et al. [33], when Ip and Martin [9] used the GHQ-12 on Chinese women in the perinatal period, they found that the GHQ-12 was not suitable for use in this clinical group. Uncertainties about the use of the GHQ-12 in the perinatal population have been expressed in earlier studies in the UK by Martin and Jomeen [34]. The GHQ-12 was designed for screening of non-psychiatric disturbances in different clinical groups, including perinatal groups. As mentioned above, the study conducted in Hong Kong by Lee et al. [33] has established that while the GHQ-12 is an admirable measure of psychological distress in many clinical populations, other studies have found the GHQ-12 to be unsuitable for use in the perinatal period [9] [34]. 
Martin and Jomeen [34] found variations in detecting cases of psychological distress by the GHQ-12, which have been attributed to differences in scoring methods. In addition, the inclusion of a somatic subscale is also said to contribute to the unsuitability of the GHQ-12 for the perinatal period because of the reduction of its discriminatory ability and increased potential to produce a positive response due to either physical or psychological disorder [34]. The above points may be possible explanations for the difference in scores between the EPDS and the GHQ-12 observed in the postnatal period.

It is documented that both the EPDS and the GHQ-12 are measures of depression and psychological distress that have been used widely. Factor analysis and validity issues have been debated by researchers specialised in the area and have been found to be appropriate for use in different cultures including those of developing countries like Zambia. However, it is acknowledged that the existence of cultural differences in the expression of distress points to the importance of developing measures that are culturally appropriate [35]. Therefore, it is pertinent to acknowledge the use of already established measures of psychological distress as a limitation in this paper, but it is also relevant to recognised that developing a measure can be time consuming as well as an expensive undertaking.

In addition to the statistically significant association between the EPDS and the GHQ-12, the results revealed that the EPDS can significantly predict psychological distress in the postnatal period using antenatal data. This finding supports Merfer et al. [36] who tested the ability of the EPDS used in pregnancy to predict postnatal depression. They assessed 1620 pregnant women from the general population and found that the mean EPDS scores were significantly higher during pregnancy in women who experienced postpartum depressive symptoms than in those without postpartum symptoms $(\mathrm{P}<0.001)$.

Similarly, it is recognized that antenatal depression is a risk factor for postnatal depression as revealed by Nhiwatiwa et al. [37], and Najman et al. [38] in their respective studies that examined some of the risk factors associated with the development of postnatal depression in mothers who regularly attended an antenatal clinic at a tertiary health care centre. Their findings show that 44 percent of women with antenatal psychological distress developed postnatal psychological distress.

Conversely, Meijer et al. [39] found that the EPDS was not sufficiently accurate in predicting risk of postpartum depressive symptoms when the commonly used cut-off score of 13 is adopted. The sensitivity was low (17\%) and if this cut-off score (13) was used, only 16.8 percent of the women would be identified as more likely to go on to develop postpartum depression. In their study, the positive predictive value was also low $(41.8 \%)$, indicating that less than half of the women who screened positive during pregnancy would go on to have Postpartum Depression. However, using 5 as the cut-off value may suffice for initial screening, which should be followed by further assessments and possibly ante- 
natal intervention when the woman scores positive. Further, when negative, women may be reassured that postpartum depressive symptoms are unlikely. On the other hand, it may also be argued that using a lower cut-off value (5), increased the sensitivity $(70.8 \%)$ but the positive predictive value was unacceptably reduced (15.9\%), and it was noted, that the negative predictive value was exceedingly high at 96.0 percent.

Additionally, Austin and Lumley [40] conducted a systematic review involving studies which combined a study-specific questionnaire with a common self report measure such as the General Health Questionnaire, the Beck Depression Inventory, or the Edinburgh Postnatal Depression Scale. They established that the proportion of women identified as "at risk" of postnatal depression during pregnancy ranged between $10-67$ percent while the actual prevalence of postnatal depression ranged between 5.5 - 31.5 percent. It is worth noting that this kind of results were attributable to the fact that most of the antenatal tools in this review were found to have low specificity and sensitivity for predicting postnatal depression.

Meijer et al. [39] further state that using the EPDS to screen for symptoms of psychological distress within the first week following delivery suggest that the EPDS may be used to predict which women may develop postnatal psychological distress and that women whose scores were 9 or above on the EPDS were about 30 times as likely to develop postnatal psychological distress within four weeks than women with lower EPDS score. In addition, Telssedre and Chabrol [41] found the EPDS useful in detecting postnatal psychological distress when administered a few days post-delivery.

\section{Conclusion}

The mental health of women during pregnancy is a neglected public health concern. Most of the mental health interventions are targeted at the postnatal period, and yet this study results support previous research evidence that emphasise the need to identify women who may be vulnerable to postnatal mental distress before delivery, in order to reduce the vulnerability to postnatal psychological distress. Interventions focused on antenatal psychological distress may reduce the severity and incidence of postnatal psychological distress and promote the psychological well-being of the mothers, their babies and the family at large. Such interventions may include intensive perinatal support provided by health professional or prophylactic antidepressants where appropriate. Therefore, integration of mental health into mainstream maternal health services is likely to facilitate identification of mothers with antenatal psychological distress so that supportive interventions are provided throughout the perinatal period to safeguard the well-being of the mother, the infant and the family at large.

\section{Study Limitations}

The authors acknowledge that the study was health facility based and thus missed 
out women who did not report to healthcare facilities. Hence recommend exploring the feasibility of community screening of postnatal mental distress in future. Secondly, our results may not necessarily extrapolate to other women within Zambia due to cultural and socioeconomic variations.

\section{Disclosure Statement}

The authors declare that there are no competing interests.

\section{Acknowledgements}

The authors wish to sincerely thank all the women who participated in this study for their time and patience.

\section{Conflicts of Interest}

The authors declare no conflicts of interest regarding the publication of this paper.

\section{References}

[1] Kessler, R.C., Berglund, P., Demler, O., Jin. R., Koretz, D., Merikangas, K.R., Rush, A.J., Walters, E.E. and Wang, P. (2003) The Epidemiology of Major Depressive Disorder: Results from the National Comorbidity Survey. JAMA, 289, 3095-105. https://doi.org/10.1001/jama.289.23.3095

[2] Adeponle, A., Groleau, D., Kola, L., Kirmayer, L.J. and Gureje, O. (2017) Perinatal Depression in Nigeria: Perspectives of Women, Family Caregivers and Health Care Providers. International Journal of Mental Health Systems, 11, 27. https://doi.org/10.1186/s13033-017-0134-6

[3] Murphey, C., Carter, P., Price, L.R., Champion, J.D. and Nichols, F. (2017) Psychological Distress in Healthy Low-Risk First-Time Mothers during the Postpartum Period: An Exploratory Study. Nursing Research and Practice, 2017, Article ID: 8415083. https://doi.org/10.1155/2017/8415083

[4] Wishman, M.A., Weinstock, L.M. and Tolejko, N. (2006) Marriage and Depression. In: Keyes, C.L.M. and Goodman, S.H., Eds., Women and Depression: A Handbook for the Social, Behavioral, and Biomedical Sciences, Cambridge University Press, New York, 219-240. https://doi.org/10.1017/CBO9780511841262.012

[5] Clarke, K., Saville, N., Shrestha, B., Costello, A., King, M., Manandhar, D.O. and Prost, A. (2014) Predictors of Psychological Distress among Mothers in Rural Nepal: A Cross-Sectional Community Based Study. Journal of Affective Disorders, 156, 76-86. https://doi.org/10.1016/j.jad.2013.11.018

[6] Mwape, L., Mukwato, P.K. and Mweemba, P. (2016) Psychological Distress during the Perinatal Period: Using Edinburgh Postnatal Depression Scale (EPDS) General Health Questionnaire (GHQ-12) and the Study Specific Measure of Perinatal Distress (SSMPD) on a Zambian Cohort. Journal of Preventive and Rehabilitative Medicine, 1, 44-52.

[7] Clarke, K., Saville, N., Shrestha, B., Costello, A., King, M., Manandhar, D.O. and Prost, A. (2014) Predictors of Psychological Distress among Mothers in Rural Nepal: A Cross-Sectional Community Based Study. Journal of Affective Disorders, 156, 76-86. https://doi.org/10.1016/j.jad.2013.11.018

[8] Elisei, S., Lucarini, E., Murgia, N., Ferranti, L. and Luigi, A. (2013) Perinatal De- 
pression: A Study of Prevalence and of Risk and Protective Factors. Psychatria Danubina, 25, 258-262.

[9] Ip, W.Y. and Martin, C.R. (2006) Factor Structure of the Chinese Version of the 12-Item General Health Questionnaire (GHQ-12) in Pregnancy. Journal of Reproductive and Infant Psychology, 24, 87-98. https://doi.org/10.1080/02646830600643882

[10] Honikman, S. and Field, S. (2008) Perinatal Mental Health Project: Caring for Mothers, Caring for the Future. South Africa. University of Cape Town.

[11] O’Hara, M. and Swan, A. (1996) Rates and Risks of Postpartum Depression-A Meta-Analysis. International Review of Psychiatry, 8, 37-54. https://doi.org/10.3109/09540269609037816

[12] Sharma, V., Burt, V.K. and Ritchie, H.L. (2009) Bipolar II Postpartum Depression, Diagnosis, and Treatment. The American Journal of Psychiatry, 166, 1217-1221. https://doi.org/10.1176/appi.ajp.2009.08121902

[13] Hegde, S., Latha, K.S., Bhat, S.M., Sharma, P.S.V.N. and Kamath, A. (2012) Postpartum Depression: Prevalence and Associated Factors among Women in India. Journal of Women's Health Issues, \& Care, 1, 1.

[14] Milgrom, J., Ericksen, J., McCarthy, R. and Gemmill, A.W. (2006) Stressful Impact of Depression on Early Mother-Infant Relations. Stress and Health, 22, 229-238. https://doi.org/10.1002/smi.1101

[15] Coverdale, J.H., McCullough, L.B., Chervenak, F.A. and Bayer, T. (1996) Clinical Implications and Management Strategies When Depression Occurs during Pregnancy. Australian and New Zealand Journal of Obstetric Gynaecology, 36, 424-429. https://doi.org/10.1111/j.1479-828X.1996.tb02185.x

[16] Parsons, C.E., Young, K.S., Rochat, T.J., Kringelbach, M.L. and Stein, A. (2012) Postnatal Depression and Its Effects on Child Development: A Review of Evidence from Low- and Middle-Income Countries. British Medical Bulleting, 101, 57-79. https://doi.org/10.1093/bmb/ldr047

[17] Surkan, P.J., Kennedy, C.E., Hurley, K.M. and Black, M.M. (2011) Maternal Depression and Early Childhood Growth in Developing Countries: Systematic Review and Meta-Analysis. Bulletin World Health Organisation, 287, 607-615. https://doi.org/10.2471/BLT.11.088187

[18] Murray, L., Cooper, P.J., Wilson, A. and Romaniuk, H. (2003) Controlled Trial of the Short- and Long-Term Effect of Psychological Treatment of Post-Partum Depression 1. Impact on Maternal Mood. British Journal of Psychiatry, 182, 420-427. https://doi.org/10.1192/bjp.182.5.420

[19] Räisänen, S., Lehto, S.M., Nielsen, H.S., Gissler, M., Kramer, M.R. and Heinonen, S. (2013) Fear of Childbirth Predicts Postpartum Depression: A Population-Based Analysis of 511422 Singleton Births in Finland. British Medical Journal Open, 3, e004047. https://doi.org/10.1136/bmjopen-2013-004047

[20] Cox, J.L., Holden, J.M. and Sagovsky, R. (1987) Detection of Postnatal Depression. Development of the 10-Item Edinburgh Postnatal Depression Scale. British Journal of Psychiatry, 150, 782-786. https://doi.org/10.1192/bjp.150.6.782

[21] Jomeen, J. and Martin, C.R. (2005) Confirming of an Occluded Component within the Edinburgh Postnatal Depression Scale (EPDS) during Pregnancy. Journal of Reproductive and Infant Psychology, 23, 143-154. https://doi.org/10.1080/02646830500129297

[22] Eberhard-Gran, M., Eskild, A., Tambs, K., Opjordsmoen, S. and Samuelsen, S.O. (2001) Review of Validation Studies of the Edinburgh Postnatal Depression Scale. 
Acta Psychiatrica Scandinavica, 104, 243-249.

https://doi.org/10.1034/j.1600-0447.2001.00187.x

[23] Brouwers, E.P., Van Baar, A.L. and Pop, V.J. (2001) Does the Edinburgh Depression Scale Measure Anxiety? Journal of Psychosomatic Research, 51, 659-663.

https://doi.org/10.1016/S0022-3999(01)00245-8

[24] Ross, L.E., Gilbert Evans, S.E., Sellers, E.M. and Romach, M.K. (2003) Measurement Issues in Postnatal Depression, Part 1: Anxiety as Feature of Postpartum Depression. Archives of Women's Mental Health, 6, 51-57. https://doi.org/10.1007/s00737-002-0155-1

[25] Goldberg, D.P. and William, P. (1988) A User's Guide to the General Health Questionnaire. NFER-Nelson, Basingstoke.

[26] Goldberg, D.P., Gater, R., Sartorius, N., Ustun, T.B., Piccinelli, M., Gureje, O. and Rutter, C. (1997) The Validation of Two Versions of the General Health Questionnaire in the WHO Study of Mental Illness in General Health Care. Psychological Medicine, 27, 191-197. https://doi.org/10.1017/S0033291796004242

[27] Mofrad, S., Abdullah, R., Abu Samah, B., Mansor, M.B. and Baba, M.B. (2009) Maternal Psychological Distress and Separation Anxiety Disorder in Children. European Journal of Social Sciences, 8, 231-349.

[28] Central Statistical Office (2014-2015) Zambia Demographic and Health Survey. CSO, Lusaka.

[29] Polit, D.F. and Beck, C.T. (2012) Nursing Research Generating and Assessing Evidence for Nursing Practice. 9th Edition, Lippincott Williams \& Wilkins, Philadelphia.

[30] Elliot, S.A. (2000) Report on the Santra Workshop on Classification of Postnatal Mental Disorders. Archives of Women's Mental Health, 3, 27-33.

[31] Cox, J.L., Chapman, G., Murray, D. and Jones, D. (1996) Validation of the Edinburgh Postnatal Depression Scale in Non-Postnatal Women. Journal of Affective Disorders, 39, 185-189. https://doi.org/10.1016/0165-0327(96)00008-0

[32] Werneke, U., Goldberg, D.P., Yalcin, I. and Ustun, B.T. (2000) The Stability of the Factor Structure of the General Health Questionnaire. Psychological Medicine, 30, 823-829. https://doi.org/10.1017/S0033291799002287

[33] Lee, D.T., Yip, A.S., Chiu, H.F., Leung, T.Y. and Chung, T.K. (2001) Screening for Postnatal Depression, Are Specific Instruments Mandatory? Journal of Affective Disorders, 63, 391-401. https://doi.org/10.1016/S0165-0327(00)00193-2

[34] Martin, C.R. and Jomeen, J. (2003) Is the 12 item General Health Questionnaire (GHQ-12) Confounded by Scoring Method during and Following Birth. Journal of Reproductive and Infant Psychology, 21, 267-278. https://doi.org/10.1080/02646830310001622088

[35] Hunt, S.M., Alonso, J., Bucquet, D., Niero, M., Wiklund, I. and McKenna, S. (1991) Cross Cultural Adaptation of Health Measures. Health Policy, 19, 33-44. https://doi.org/10.1016/0168-8510(91)90072-6

[36] Meijer, J., Beijers, C., van Pampus, M., Verbeek, T., Stolk, R.P., Milgrom, J., Bockting, C.L.H. and Burger, H. (2014) Predictive Accuracy of Edinburgh Postnatal Depression Scale Assessment during Pregnancy for the Risk of Developing Postpartum Depressive Symptoms: A Prospective Cohort Study. Epidemiology, 121, 1604-1610.

[37] Nhiwatiwa, S., Patel, V. and Acuda, W. (1998) Predicting Postnatal Mental Disorder with a Screening Questionnaire: A Prospective Cohort Study from Zimbabwe. Journal of Epidemiology \& Community Health, 52, 262-266. 
https://doi.org/10.1136/jech.52.4.262

[38] Najman, J.M., Williams, G.M., Nikles, J., Spence, S., Bor, W., O’Callaghan, M., LeBrocque, R. and Andersen, M.J. (2000) Mothers' Mental Illness and Child Behavior Problems: Cause-Effect Association or Observation Bias? Journal of the American Academy of Child and Adolescent Psychiatry, 39, 592-602.

https://doi.org/10.1097/00004583-200005000-00013

[39] Meijer, J.L., Beijers, C., van Pampers, M.G., Verbeek, T., Stolk, R.P., Milgrom, J., Bockting, C.L.H. and Burger, H. (2014) Predictive Accuracy of the Edinburgh Postnatal Depression Scale Assessment during Pregnancy for the Risk of Developing Postnatal Depressive Symptoms: A Prospective Cohort Study. International Journal of Obstetrics and Gynaecology, 121, 1604-1610.

https://doi.org/10.1111/1471-0528.12759

[40] Austin, M.P. and Lumley, J. (2000) Antenatal Screening for Postnatal Depression: Systematic Review. Acta Psychiatrica Scandinavica, 107, 10-17.

https://doi.org/10.1034/j.1600-0447.2003.02024.x

[41] Telssedre, F. and Chabrol, H. (2004) A Study of the Edinburgh Postnatal Depression Scale (EPDS) on 859 Mothers: Detection of Mothers at Risk for Postpartum Depression. Encephale, 30, 376-381. 